\title{
Resolution of Blind Defects in Metallic Plate under Uniform Heating
}

\section{Guohua LI, Huijuan WEI, Hualong LI, Miao WU}

School of Mechanical, Electronic \& Information Engineering, China University of Mining and Technology, Beijing (CUMTB),D11,Xueyuan Road, Haidian District, Beijing, China, 100083

\author{
lgh@cumtb.edu.cn
}

In order to explore the resolution of blind defects in metallic plates by infrared thermography under stimulation of uniform heating, both simulative and experimental approaches are carried out. 3D simulative models of metallic plates with blind defects of different size at different depth are created to do finite element analysis, and then preliminary experimental study are done to verify the simulation result. In the simulation, both single metallic plates and metal/metal composite plates are detected, and inference of several factors such as the thickness of coating and matrix, the thermal properties of coating and matrix, the stimulating intensity and detecting method(reflection or transmission) on the side of coating or matrix are considered. In the preliminary experimental study, steel and aluminum samples with inner defects are manufactured, and a simple test rig is designed.

Keywords: Resolution, Blind defects, Metallic plate, Uniform Heating, Simulation, Experiment 